\title{
Influencia del perfil de los jóvenes en sus valores del cuerpo Influence of the profile of young people on their body values
}

\section{*LorenaVelasco-Santos, **J osé Luis Pastor Pradillo, ***David Blanco-Alcántara, ***Alfredo Jiménez Eguizábal} *Educación Física en Secundaria (España), **U niver sidad deAlcalá (España), *** Universidad de Burgos (España)

\begin{abstract}
Resumen. El presente artículo analiza los valores del cuerpo de 536 estudiantes de 1o Bachillerato en Castilla y León en el curso 2017/ 18 en función de sus variables de perfil sexo, modalidad de bachillerato, natural eza, titularidad y provincia de pertenencia del centro de estudios. El objetivo es describir tendencias generales asociadas a dichas variables de perfil. El instrumento de medida es el test elaborado por Casares y Collados (1998) que clasifica en 10 los valores del cuerpo en base a 250 ítems agrupados en bloques de 25. El tratamiento de datos se lleva a cabo mediante un análisis de val ores medios. Los resultados obtenidos son mostrados en formade tablas de puntuaciones y representacionesjerárquicas. Se cal culan estadísticos descriptivosy estadísticainferencial. Se observan diferencias estadísticamente significativas asociadas al a variable de perfil sexo y, secundariamente, provincia de pertenencia del centro de estudios. Asimismo, destacael valor placer como el másagradable en todas las clasificaciones y el valor religioso el menos, exceptuando lo ocurrido en Soria donde se torna incluso positivo. Todo ello describe la realidad en que los estudiantes de 1 - bachillerato conciben su cuerpo y le conceden su val or, lo que abre nuevos flancos críticos en la educación a través de los val ores del cuerpo.
\end{abstract}

Palabras clave. Variables de perfil, sexo, modalidad de bachillerato, naturaleza, titularidad, provincia.

\begin{abstract}
This article analyzes the body values of 536 FirstYear Bachillerato students in the Castilla y León region of Spain during the academic year 2017/ 18 according to their special characteristics such as gender, Bachillerato option, personality, qualifications and province administering the educational establishment they attend. The aim is to describe general trends associated with these profile variables. The instrument of measurement is the test devised by Casares and Collados (1998), which divides body val ues into 10 categories. It is based on 250 items classified into 10 groups of 25 defining the body values. Data processing is carried out through an analysis of average values. The results obtained are shown in the form of score tables and hierarchical representations. Descriptivestatistics and inferential statisticsare calculated. Significant differences associated to the variable of gender profile and, secondarily, province of belonging to the study center are highlighted. Likewise, the pleasure value stands out as the most pleasant in all classifications and the religious value the least, except for what happened in Soria where it becomes even positive. The procedure of preferred val ues ratifies the pleasure value as the most pleasant, however the religious value is rarely the least preferred. All this describes the reality in which the students of the 1st baccalaureate conceive their body and give it their value, which opens new critical flanks in education through the values of the body.
\end{abstract}

Keywords. Variables profile, gender, high school modality, nature, ownership, province.

\section{Introducción}

Los valores del cuerpo identifican la esencia más profunda del ser humano en su visión de la realidad y de sí mismo, y a la vez orientan su camino definiendo el rumbo que él mismo tomará en dicha realidad.

De una parte, los valores - cualidad, ideal o real, deseada o deseable por su bondad, cuya fuerza estimativa-utópica orienta la vida humana (Gervilla, 2000a, p.17)- son una construcción personal, forjados en la experiencia y el aprendizaje, e influenciados por múltiples factores que rodean al individuo 0 acontecen a lo largo de su existen-

Fecha recepción: 21-09-20. Fecha de aceptación: 24-12-20 Lorena Velasco-Santos

lorenavelascoes@yahoo.es cia y proceso de formación continua. Por otro lado, superando cualquier dualismo filosófico, el cuerpo es la dimensión humana que queda fusionada con la razón y el afecto en forma de una unidad indisociable y entera mente conectada. El cuerpo supone desde la realidad más somática y aparente al alcance del mundo tanto exterior como interior, hasta su esencia intangible emergente, y es en todas sus dimensiones al tamente influido socialmente.

El binomio valor y cuerpo alcanza su mayor vulnerabilidad en la adol escencia, momento en que se producen acelerados cambios del organismo en estructura y función que repercuten grandes cambios biológicos, psicológicos y sociales (GarcíaC aballero C., 2000). Pero, siguiendo a Fize M. (2004), este periodo de la adolescencia acusa más los cambios social es que los psico-bio- 
lógicos, puesto que el adolescente encuentra mayores dificultades en la integración al cuerpo social que al suyo propio.

Resulta complejo identificar el tipo de influencia acontecida en la formación de los valores del cuerpo de cada ser humano dentro de su haber único e irrepetible, en la medida que podrían ser asociados a una influencia externa, ya sea a nivel social más generalizado, o de un colectivo más reducido en función de la variable de perfil tenida en cuenta y su campo de implicación y repercusión, 0 val ores que podrían responder a una demanda meramente anatomo-fisiológica condicionada biológicamente por su estructura y funcionamiento de base. Sin embargo, cualquier variable de perfil, incluso las a priori definidas como mera identidad biológica, como pueda ser el sexo, quedan asociadas a su adscripción socio-cultural. Del sexo emerge toda una construcción social sobre el género y cada una de sus categorías parece vincularse a unas connotaciones y finalmente a unos valores diferenciados.

Analizar la influencia de determinadas variables de perfil en la formación de valores, al margen de poder asociar esta influencia a constructos sociales o psico-biológicos, podría facilitar el estudio y la identificación de los mismos.

Son numerosos los estudios que proceden a analizar los valores de la persona y la influencia de variables como el sexo del individuo o la tipología de estudios universitarios (Álvarez, 2002; Álvarez, Rodríguez y Lorenzo 2007; Cámara2010; Q uijano y Lorenzo 2015; Q uijano, Lorenzo y Alegre 2016), sin embargo, ningún estudio concreta el análisis en los valores del cuerpo en sí mismos o qué variables podrían condicionar tales posicionamientos axiológicos.

Surge así el interés por relatar las variables que podrían condicionar los valores del cuerpo, comenzando por las más contempladas en los estudios de los valores de la persona, es decir, el sexo y la tipología de estudios cursados, continuando por otras variables asociadas al centro de estudios como pueda ser la titularidad (pública o privada) donde la principal diferencia radica en el carácter religioso de los centros privados-concertados estudiados, cumpliendo con las particularidades descritas por autores como Faubell, V. (2000) y González, T. (2005). Por último, emerge el interés por analizar unidades mínimas que queden definidas por una identidad propia. En este sentido, se valora la provincia como unidad territorial con idiosincrasia propia, así como la variable naturaleza que diferencia el ámbito rural y el urbano. En cualquier caso, siguiendo a Giménez, G.
(2010), en consonancia con Izzo (1985), las identidades se inscriben y apoyan en un contexto de representaciones social es compartidas, es decir, tradiciones cultura les, expectativas recíprocas, saberes compartidos y esquemascomunes, en definitiva, unaestructuraaxiológica compartida. Por su parte, Inglehart (1991) sostiene que son las condiciones sociales en las que los jóvenes se crían y educan las que configuran prioritariamente su sistema de valores, y así también, de valores del cuerpo en la medida en que, como afirma Barbero (2007, p. 26) «el capital corporal forma parte del amplio y diverso conjunto de capitales cultural ess.

Como previamente se señalaba, todas las variables de perfil previamente definidas entroncan una construcción socio-cultural de los valores del cuerpo, por lo que identificar los valores asociados a cada una de las categorías descrita por cada variable podría ser muy útil en cual quier intervención en valores a todos los niveles, ya sea político, económico 0 , en este caso, educativo.

En este contexto, el presente estudio persigue identificar algunas de las variables que pueden influir en la construcción de los valores del cuerpo de los estudiantes de 10 bachillerato como muestra representativa de la sociedad occidental contemporánea y apuntar al gunas pautas pedagógicas de intervención educativa. El objetivo es así descubrir cuáles de las variables de perfil estudiadas, sexo y modalidad de bachillerato del estudiante, y naturaleza, titularidad y provincia de pertenencia de su centro de estudios, describen diferencias estadísticamente significativas en los valores del cuerpo de los estudiantes de 10 de Bachillerato, así como valorar si dicha realidad descrita por los resultados merece o no una revalorización corporal.

Y sobre la descripción objetiva de la realidad que acontece podremos sentar las bases de una oportuna intervención educativa, previa a una reflexión crítica sobre la pureza y salud que los valores destapados representan. Los valores son indisociables de cualquier existencia, son inherentes a su realidad, ignorarlos sólo podría conducir a transmitirlos sin previa intención ni capacidad de obrar sobre los mismos mediante el currículum oculto.

\section{M etodología}

\section{Participantes}

En primer lugar, se solicitó la participación de todos los centros enviando un correo electrónico a la dirección institucional de cada centro, con el interés expreso de que éste fuera reenviado al Departamento de Edu- 
cación Física y finalmente llegase al profesor al cargo de 10 de Bachillerato. Sin embargo, fueron escasos los centros que mostraron su interés por participar, de modo que se recurrió a la vía telefónica estableciendo contacto directo con cada uno de los 274 profesores responsa bles en cada centro. Finalmente fueron 60 los centros participantes. La mayoría de ellos mostraron su predilección por colaborar en formato papel y no mediante tecnologías más avanzadas, puesto que era la única ma nera de asegurar que el procedimiento pudiese ser completado por todos los estudiantes. Esto ralentizó el proceso dado que hubo que tabular posteriormente toda la información del papel al soporte digital. El proceso de recogida de datos se inició al final del segundo trimestre del curso escolar 2017/ 2018 y finalizó coincidiendo con el final del curso escolar, sin embargo, la solicitud de participación en el presente estudio comenzó al inicio del curso escolar.

En el estudio han participado 536 estudiantes matriculados en 1 - bachillerato en al guno de los 60 centros de Castilla y León participantes de los 274 registrados en la comunidad autónoma. La población total de estudiantes es de 15.894 y la muestra ha sido escogida siguiendo dos criterios prioritariamente: asegurar la cantidad mínima para que ésta sea representativa, y que fuese porcentual a cada una de las categorías resultantes de la aplicación de las variables estudiadas.

En cuanto a la cantidad se ha asegurado superar la muestra mínima establecida para poblaciones finitas (tamaño inferior a 100.000 elementos) según muestreo aleatorio simple definido por la Ecuación 1.

$$
n=\frac{Z 2 \times N \times p \times q}{E 2 \times(N-1)+Z 2 \times p \times p}
$$

donde:

$\mathrm{n}=$ tamaño de la muestra que se quiere calcular.

$Z$ = desviación del valor medio que se acepta para lograr el nivel de confianza deseado.

$\mathrm{N}=$ tamaño de la población o universo.

$p=$ proporción de la población que posee la característica objeto de estudio.

$q=1-p$.

$\mathrm{E}=$ el error máximo admitido.

Partiendo de la fórmula expuesta, se concretan a continuación los parámetros de la misma para este tra bajo de investigación. Así, el nivel de confianza se esta blece en un $95 \%$, valor que conduce a un parámetro $\mathrm{Z}=1,96$. Respecto al tamaño de la población (N), asciendea 15.894 estudiantesen el curso 2017-18. Laproporción de la población que posee la característica obje- to de estudio no es conocida, luego se aplica el valor que exige un mayor tamaño de muestra para garantizar el proceso, siendo este valor de 0,5 . Consecuentemente, el parámetro q es 0,5 resultado de la ecuación (1-p). Y, finalmente, el error máximo admitido es del $5 \%$.

A plicando estos valores a la fórmula expresada anteriormente para el cálculo al eatorio simple con pobla ciones finitas, al tratarse de una población menor a 100.000, se obtiene:

$$
n=\frac{1.962 \times 15894 \times 0.5 \times 0.5}{0.052 \times(15894-1)+1.962 \times 0.5 \times 0.5}
$$

Esta cifra de 376 individuos (El número de individuos encuestados, y por tanto de cuestionarios, no admite decimales. De forma, que en estos casos se redondeará al número entero inmediato superior), en términos porcentuales, representa cerca de un 2,40\% de la población total.

En cuanto a la distribución de la muestra, se ha pretendido mantener los porcentajes descritos por la población total en función de las cinco variables de perfil analizadas: sexo y modalidad de bachillerato del alumno, y naturaleza, titularidad y provincia de pertenencia del centro de estudios.

De este modo, y con la advertida intención de cubrir la muestra mínima calculada y su distribución proporcional, se ha aplicado un 2,50\% sobre cada una de las categorías resultantes de la combinación de variables. Sin embargo, la muestra disponible limita esta aspira ción hipotética o muestra teórica, teniendo que compensar la carencia de al gunas categorías cruzadas de cinco variables para asegurar las posibles combinaciones de hasta cuatro variables, resultando finalmente una muestra de 536 estudiantes distribuidos según sus diferentes variables de perfil como se indica en laTabla 1. En la primera columna se advierte la variable de perfil considerada, en la segunda las categorías en que dicha

\begin{tabular}{|c|c|c|c|c|}
\hline & ables & Población & Muestra & $\begin{array}{c}\% \\
\text { Población }\end{array}$ \\
\hline \multirow{2}{*}{ Sexo } & Hombres & 7.598 & 254 & $3,34 \%$ \\
\hline & Mujeres & 8.296 & 382 & $3,40 \%$ \\
\hline \multirow{3}{*}{ Modalidad } & Ciencias & 7.714 & 259 & $3,36 \%$ \\
\hline & HH y CC SS & 7.390 & 251 & $3,40 \%$ \\
\hline & Artes & 786 & 26 & $3,31 \%$ \\
\hline \multirow{2}{*}{$N$ aturaleza } & Urbano & 11.205 & 367 & $3,28 \%$ \\
\hline & Rural & 4.689 & 169 & $3,60 \%$ \\
\hline \multirow{2}{*}{ Titularidad } & Público & 12.307 & 412 & $3,35 \%$ \\
\hline & Privado-concertado & 3.587 & 124 & $3,46 \%$ \\
\hline \multirow{9}{*}{ Provincia } & Ávila & 1.060 & 32 & $3,02 \%$ \\
\hline & Burgos & 2.331 & 119 & $5,11 \%$ \\
\hline & León & 3.068 & 86 & $2,80 \%$ \\
\hline & Palencia & 973 & 33 & $3,39 \%$ \\
\hline & Salamanca & 2.308 & 81 & $3,51 \%$ \\
\hline & Segovia & 1.040 & 34 & $3,27 \%$ \\
\hline & Soria & 612 & 24 & $3,92 \%$ \\
\hline & Valladolid & 3.377 & 94 & $2,78 \%$ \\
\hline & Zamora & 1.125 & 33 & $2,93 \%$ \\
\hline Total & Total & 15.894 & 536 & $3,37 \%$ \\
\hline
\end{tabular}
variable resulta, en la tercera la población censada en 
dicha categoría, en la cuarta la muestra finalmente tenida en cuenta y en la quinta el porcentaje sobre la población total que dicha muestra representa.

En primer lugar, se pidió consentimiento expreso a laConsejeríade Educación de laJ unta de Castillay León, en segundo lugar, al director de cada uno de los centros participantes, en tercer lugar, al profesor de Educación Física y finalmente a cada uno de los alumnos participantes. No obstante, los datos se han tratado en todo momento de forma anónima.

\section{Instrumento}

El instrumento de medida empleado en la presente investigación ha sido el test de valores del cuerpo ela borado por Casares y Collados (1998). Este test pretende analizar los valores otorgados al cuerpo por el individuo, para lo cual se proponen 10 categorías, sentidos 0 valores del cuerpo diferenciados: 1. Biológico, 2. Ecológico, 3. Instrumental, 4. Dinámico, 5. Afectivosocial, 6. Ético, 7. Estético, 8. Religioso, 9. Intelectual y, por último, 10. Placer.

Cabe señalar que este instrumento de medida es una forma indirecta de poder obtener una puntuación cuantitativa de los valores del cuerpo. El val or que un individuo pueda dar a su cuerpo es una variable no observable, por lo que se hace necesario recurrir a un mecanismo que ayude y contribuya a obtener una medida mesurable de la misma.

El fundamento de este test reside en la reacción valorativa de agrado, desagrado 0 indiferencia experimentada al leer 0 escuchar al gunas palabras, donde el individuo debe indicar el tipo e intensidad de su reacción. En total consta de 250 palabras-expresiones agrupadas en bloques de 25 palabras, donde cada bloque se corresponde con una de las 10 categorías o sentidos de valor del cuerpo previamente mencionados.

Siguiendo a Gervilla (2000aㅡ, p. 219-220), se detalla la connotación asumida para cada uno de los 10 valores del cuerpo, anunciando entre paréntesis los 25 ítems que el autor dedica a cada una de las 10 categorías de cuerpo: 1. Biológico (1-25): valores referidos a su ma teria viva, conservación, desarrollo y cuidado material; 2. Ecológico (26-50): valores como parte de la natura leza, receptor de los bienes de su entorno natural, referentes a su relación con ella; 3. Instrumental (51-75): valores como medio para realizar tareas diversas, así como para construir, modificar o utilizar todo tipo de materiales u objetos con finalidad práctica y eficaz; 4. Dinámico (76-100): valores relativos, prioritariamente, al cuerpo en movimiento y a sus posibilidades materia- lizadas en actos perceptibles, con un sentido lúdico, deportivo, de ocio, etc.; 5. Afectivo-social (101-125): va lores como expresión de sentimientos, estados de ánimo y/ o formas de comunicación con los demás; 6 . Ético (126-150): valores relacionados con la bondad o malicia de los actos humanos, en relación consigo mismo y con los demás; 7. Estético (151-175): valores relacionados con la percepción, creación y expresión de la belleza y el arte, en el cuerpo y mediante el cuerpo; 8. Religioso (176-200): valores relacionados directamente con actividades y modos de expresión corporales relativos a la fe y las creencias religiosas; 9. Intelectual (201-225): valores relacionados prioritariamente con la base corporal de las funciones cognitivas e intelectuales del sujeto, así como con aquellas actividades perceptibles en las que el cuerpo interviene para adquirir o expresar conocimientos; 10. Placer (226-250): valores relaciona dos con el cuerpo en cuanto nos proporciona bienestar, satisfacción y gozo.

El test se cumplimenta por parte de los estudiantes según unas directrices. Cada una de las propuestas ha de ser calificada rodeando con un círculo o marcando con una cruz una de las al ternativas situadas a su derecha, las cuales se gradúan de más a menos agradable en cinco categorías: $M A=$ M uy agradable, $A=$ Agradable, $I=$ Indiferente, $\mathrm{D}=$ Desagradable, y $\mathrm{MD}=$ M uy desagra dable. Se marca aquella opción que se considere más representativa con la impresión o el interés que le suscite, teniendo en cuenta que losítems marcados con un asterisco deben ser calificados al revés, es decir, cuando algo fuera agradable, habría de marcarse desagradable. No deben dejarse contestaciones sin responder. No es necesario detenerse mucho tiempo para pensar la puntuación a cada término, sino que por el contrario se trata de dar una respuesta lo más intuitiva posible puesto que es esta primera idea o intuición la que se demuestra como más sincera y fiel a la realidad de valor. Es importante ajustar la intensidad de la reacción que produzca cada palabra a la escala de respuestas. No hay tiempo límite.

\section{Propiedades psicométricas}

Antes de comenzar con los cál culos propiamente dichos, se lleva a cabo un estudio de fiabilidad para el caso concreto de la muestra objeto de estudio. Se comprueba, mediante el cálculo del alfa de Cronbach, que el instrumento es fiable para la muestra de estudiantes escogida. Este estadístico permite comprobar la consistencia interna para la escala total, concluyendo que dicho instrumento presenta adecuadas propiedades 
psicométricas. A pesar de ser el alfa de Cronbach un estadístico ampliamente utilizado en la literatura para comprobar la fiabilidad de este tipo de instrumentos de recogida de datos, cabe señalar que no existe acuerdo unánime que determine el valor exacto a partir del cual se pueda afirmar la fiabilidad del instrumento de medida. Si bien, un instrumento de medida puede considerarse fiable si muestra un valor superior a 0.7-0.8, siendo tanto más idóneo cuanto más se acerque al valor 1. En este caso, el Alfa de Cronbach toma un valor de 0,956 .

\section{Procedimiento}

La corrección del test se ha lleva a cabo según las indicaciones descritas por sus autores (Casares y Colla dos, 1998). Se separan las categorías de cuerpo en las diez dispuestas asignando a cada una de ellas los veinticinco ítems que se relacionan, y multiplicando cada una de las respuestas por un coeficiente en función de la calificación marcada. Las respuestas muy agradables se multiplican por +2 , las agradables por +1 , las indiferentes por 0 , las desagradables por -1 y las desagrada bles por -2. U na vez realizado este paso, se procede a la suma de las veinticinco puntuaciones obtenidas que conforman cada categoría de valor del cuerpo, siendo esta suma la puntuación final otorgada a ese val or del cuerpo del individuo. Así, esta puntuación final, deberá encontrarse en el interval o comprendido entre la puntuación máxima de +50 y mínima de -50 .

El test genera 10 valores del cuerpo resultantes de la agrupación de 25 ítems. Se cal cula la media de cada uno de estos valores en cada una de las categorías descritas por las cinco variables de perfil, sexo (hombre y mujer) y modalidad de bachillerato (Ciencias y Tecnología, Humanidades y Ciencias Sociales y Arte), y titularidad (distinguiendo entre pública y privada-concertada), natural eza (urbano o rural) y provincia de pertenencia del centro de estudios (las 9 constitutivas de la Comunidad Autónoma de Castilla y León).

El estudio muestra las puntuaciones medias obtenidas en cada uno de los valores para cada una de las categorías resultantes de la aplicación de estas cinco varia bles. Asimismo, muestra los valores de dispersión 0 desviación típica en cada caso, las puntuaciones medias para cada valor en todas las categorías según la variable considerada, y la diferencia entre las dos puntuaciones para el caso de las variables que sólo admiten dos categorías como son el sexo, la naturaleza o la titularidad. Estos valores son mostrados en forma de tablas de puntuaciones. Asimismo, se lleva a cabo una diferencia de medias que anuncia si las disparidades observadas en las puntuaciones son o no estadísticamente significativas. Finalmente, se identifican las variables de perfil que comportan cinco o más valores del cuerpo con diferencias estadísticamente significativas entre las categorías derivadas, y qué categorías obtienen mayor agrado 0 desagrado en su caso.

\section{Análisis estadístico de los datos}

Para poder desarrollar el estudio aquí presentado se trabaja con opciones avanzadas de la hoja de cál culo, así como con el programa estadístico SPSS v.23. Además de los estadísticos descriptivos se aplican procedimientos metodológicos ligados a la estadística inferencial, tales como los test de diferencia de medias, que permiten afirmar si los resultados obtenidos sobre el efecto de las variables del perfil en el grado mostrado para cada valor del cuerpo de los estudiantes son o no significativos.

Como paso previo a la aplicación de las técnicas inferenciales se analiza si las variables siguen o no una distribución normal para escoger la prueba o test adecuado. Para dicha comprobación se realiza la prueba de Kolmogorov-Smirnov de normalidad que arroja valores $Z$ con probabilidades ( $p$-valor) inferiores a 0.05 (nivel de confianza de un 95\%) en la mayoría de las variables de categorías de valores del cuerpo, por lo cual se concluye que las variables no siguen una distribución normal. De este modo, los análisis de estadística inferencial se realizan mediante técnicas no paramétricas. En concreto, se aplica la prueba U de $M$ ann-W hitney para los casos de comparación entre dos grupos y la prueba $\mathrm{H}$ de Kruskal-Wallis para los casos decomparación entremás de dosgrupos. Deestemodo, se analizasi las diferencias obtenidas son estadísticamente significativas, de forma que pueda concluirse si una determinada característica del perfil de los estudiantes influye en los valores del cuerpo obtenidos.

\section{Resultados}

En este apartado se ha analizado si las características que definen el perfil de los estudiantes conducen a evidenciar diferencias de los valores del cuerpo entre las categorías resultantes de la aplicación o división según dichas variables de perfil.

Se han analizado las puntuaciones en cada categoría según cada una de las 5 variables en lasTablas 2, 3, 4, 5 y 6 respectivamente. Asimismo, las correspondientes ta blas de puntuaciones incluyen una columna con las pun- 
tuaciones medias en cada valor del cuerpo aunando todas las categorías según dicha variable. Las varia bles que resultan en dos categorías (sexo, naturaleza y titularidad) introducen otra columna con la diferencia de las puntuaciones en cada val or en las dos categorías. La última columna de estas 5 tablas muestra los resultados de la diferencia de medias advirtiendo tres $* * *$ cuando las diferencias son estadísticamente significativas con valores de hasta 0.03 , dos** paravalores de hasta 0.05 y una* cuando no llegan a poder ser consideradas estadísticamente significativas, pero están muy cerca de poder serlo, valores de hasta 0.08 .

Tabla 2.

\begin{tabular}{|c|c|c|c|c|c|}
\hline Valores & Hombres & Mujeres & Total general & $\begin{array}{c}\text { Diferencia } \\
\text { (Hom-M uj) }\end{array}$ & Test M edias \\
\hline Biológico & $25,22(8,50)$ & $25,07(7,99)$ & $25,14(8,23)$ & 0,15 & \\
\hline Ecológico & $12,33(12,47)$ & $11,38(10,26)$ & $11,83(11,36)$ & 0,95 & \\
\hline Instrumental & $9,46(9,49)$ & $7,63(7,99)$ & $8,50(8,77)$ & 1,83 & ** \\
\hline Dinámico & $21,53(11,15)$ & $20,01(11,21)$ & $20,73(11,20)$ & 1,52 & * \\
\hline 5. Afectivo/ social & $22,74(10,67)$ & $26,84(10,22)$ & $24,90(10,63)$ & $-4,10$ & $* * *$ \\
\hline 6. Ético & $14,98(9,12)$ & $16,76(7,91)$ & $15,92(8,54)$ & $-1,78$ & *** \\
\hline 7. Estético & $21,19(12,62)$ & $27,04(12,38)$ & $24,26(12,82)$ & $-5,85$ & *** \\
\hline 8. Religioso & $-8,21(21,63)$ & $-4,45(19,00)$ & $-6,23(20,36)$ & $-3,76$ & ** \\
\hline 9. Intelectual & $24,59(13,13)$ & $24,40(11,56)$ & $24,49(12,32)$ & 0,19 & \\
\hline 10. Placer & $33,00(11,82)$ & $34,40(10,95)$ & $33,73(11,38)$ & $-1,40$ & \\
\hline
\end{tabular}

Fuente: Elaboración propia

Tabla 3.

Puntuaciones de los valores del cuerpo según sus valores medios en función de la variable modalidad de bachillerato.

\begin{tabular}{lccccc}
\multicolumn{1}{c}{ Valores } & Artes & $\begin{array}{c}\text { Ciencias y } \\
\text { Tecnología }\end{array}$ & $\begin{array}{c}\text { Humanidades y } \\
\text { Ciencias Sociales }\end{array}$ & Total general & Test medias \\
\hline 1. Biológico & $23,08(10,41)$ & $25,04(7,71)$ & $25,46(8,49)$ & $25,14(8,23)$ & \\
2. Ecológico & $12,58(13,42)$ & $12,17(10,66)$ & $11,39(11,86)$ & $11,83(11,36)$ & \\
3. Instrumental & $9,62(10,55)$ & $8,65(8,52)$ & $8,23(8,85)$ & $8,50(8,77)$ & \\
4. Dinámico & $19,08(13,47)$ & $22,09(11,12)$ & $19,50(10,90)$ & $20,73(11,20)$ & $* *$ \\
5. Afectivo/ social & $21,23(12,88)$ & $24,89(10,27)$ & $25,29(10,71)$ & $24,90(10,63)$ & \\
6. Ético & $13,19(9,42)$ & $15,49(8,33)$ & $16,64(8,61)$ & $15,92(8,54)$ & \\
7. Estético & $20,65(14,52)$ & $24,28(12,98)$ & $24,63(12,46)$ & $24,26(12,82)$ & \\
8. Religioso & $-10,88(20,02)$ & $-6,07(20,97)$ & $-5,92(19,76)$ & $-6,23(20,36)$ & \\
9. Intelectual & $23,88(16,00)$ & $25,49(11,93)$ & $23,52(12,25)$ & $24,49(12,32)$ & \\
10. Placer & $31,92(15,15)$ & $34,40(10,52)$ & $33,23(11,79)$ & $33,73(11,38)$ & \\
Fuente: Elaboración propia. & & & &
\end{tabular}

Tabla 4.

Puntuaciones de los valores del cuerpo según sus valores medios en función de la variable naturaleza del centro de estudios.

\begin{tabular}{lccccc}
\hline \multicolumn{1}{c}{ Valores } & Urbano & Rural & Total general & $\begin{array}{c}\text { Diferencia } \\
\text { (Ur-Ru) }\end{array}$ & Test medias \\
\hline 1. Biológico & $25,12(8,14)$ & $25,20(8,43)$ & $25,14(8,23)$ & $-0,08$ & \\
2. Ecológico & $11,76(11,53)$ & $11,98(11,01)$ & $11,83(11,36)$ & $-0,22$ & \\
3. Instrumental & $8,11(8,77)$ & $9,35(8,74)$ & $8,50(8,77)$ & $-1,24$ & \\
4. Dinámico & $21,30(11,11)$ & $19,49(11,32)$ & $20,73(11,20)$ & 1,81 & \\
5. Afectivo/ social & $25,15(10,69)$ & $24,35(10,50)$ & $24,90(10,63)$ & 0,80 & \\
6. Ético & $15,79(8,74)$ & $16,19(8,11)$ & $15,92(8,54)$ & $-0,40$ & \\
7. Estético & $24,37(12,25)$ & $24,05(14,01)$ & $24,26(12,82)$ & 0,32 & \\
8. Religioso & $-5,17(20,86)$ & $-8,52(19,08)$ & $-6,23(20,36)$ & 3,35 & $*$ \\
9. Intelectual & $23,99(12,36)$ & $25,56(12,19)$ & $24,49(12,32)$ & $-1,57$ & $*$ \\
10. Placer & $34,08(10,77)$ & $32,97(12,61)$ & $33,73(11,38)$ & 1,11 & \\
\hline Fuente: Elaboración propia & & & & \\
\end{tabular}

Tabla 5

Puntuaciones de los valores del cuerpo según sus valores medios en función de la variable titularidad del centro de estudios.

\begin{tabular}{lccccc}
\multicolumn{1}{c}{ Valores } & Público & $\begin{array}{c}\text { Privado- } \\
\text { Concertado }\end{array}$ & Total general & $\begin{array}{c}\text { Diferencia } \\
\text { (Pu-Pv) }\end{array}$ & $\begin{array}{c}\text { Test } \\
\text { medias }\end{array}$ \\
\hline 1. Biológico & $25,02(8,34)$ & $25,53(7,86)$ & $25,14(8,23)$ & $-0,51$ & \\
2. Ecológico & $11,58(11,62)$ & $12,65(10,46)$ & $11,83(11,36)$ & $-1,07$ & \\
3. Instrumental & $8,42(8,81)$ & $8,74(8,69)$ & $8,50(8,77)$ & $-0,32$ & \\
4. Dinámico & $20,44(11,25)$ & $21,69(11,02)$ & $20,73(11,20)$ & $-1,25$ & \\
5. Afectivo/ social & $24,93(10,72)$ & $24,79(10,34)$ & $24,90(10,63)$ & 0,14 & \\
6. Ético & $15,67(8,71)$ & $16,75(7,94)$ & $15,92(8,54)$ & $-1,08$ & \\
7. Estético & $23,95(12,76)$ & $25,32(13,03)$ & $24,26(12,82)$ & $-1,37$ & $* *$ \\
8. Religioso & $-7,71(20,32)$ & $-1,31(19,77)$ & $-6,23(20,36)$ & $-6,40$ & $* * *$ \\
9. Intelectual & $24,74(12,31)$ & $23,65(12,36)$ & $24,49(12,32)$ & 1,09 & \\
10. Placer & $33,83(11,46)$ & $33,41(11,18)$ & $33,73(11,38)$ & 0,42 & \\
Fuente: Elaboración propia. & & & &
\end{tabular}

Tabla 6.

Puntuaciones de los valores del cuer po según sus valores medios en función de la variable provincia. Valores Ávila Burgo León Palen Salam Segov Soria Vallad Zamo Total Test

$\begin{array}{lllllllllll}\text { Biológico } & 25,63 & 24,59 & 24,83 & 25,94 & 26,12 & 26,29 & 23,38 & 24,95 & 24,94 & 25,14\end{array}$ $\begin{array}{cccccccccc}(10,46) & (8,64) & (8,49) & (7,47) & (8,37) & (7,89) & (7,95) & (7,72) & (6,06) & (8,23) \\ 8,78 & 12,62 & 11,87 & 11,15 & 13,54 & 14,26 & 10,42 & 11,43 & 7,94 & 11,83\end{array} \quad *$

2. Ecológico $\quad(8,84)(11,10)(11,04)(14,06)(11,03)(12,50)(14,95)(10,82)(9,83)(11,36)$

$\begin{array}{llllllllllll}3 . & \text { Instrumental } & 10,34 & 6,70 & 9,09 & 10,24 & 9,80 & 11,18 & 6,33 & 7,43 & 8,58 & 8,50\end{array} * *$

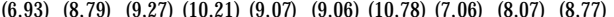

4. Dinámico $\quad \begin{array}{lllllllllll}17,47 & 22,34 & 17,93 & 20,73 & 22,94 & 21,44 & 19,54 & 21,59 & 17,70 & 20,73\end{array} \quad * * *$ $(13,33)(10,14)(11,45)(12,11)(11,49)(11,04)(13,39)(10,65)(8,34)(11,20)$

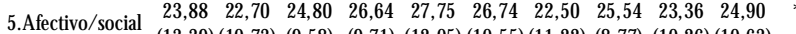
5.Afectivo/ social $(13,39)(10,73)(9,58)(9,71)(12,05)(10,55)(11,22)(8,77)(10,26)(10,63)$

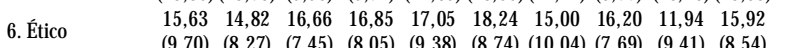

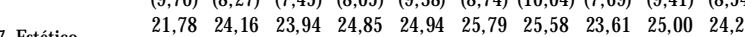
7. Estético $\quad(17,23)(13,35)(13,17)(11,70)(12,73)(13,54)(11,89)(11,02)(11,96)(12,82)$

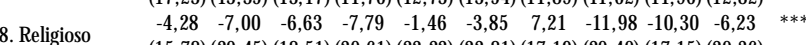
$(15,78)(20,45)(18,51)(20,61)(22,22)(22,81)(17,19)(20,40)(17,15)(20,36)$

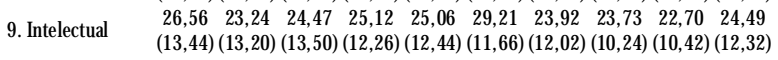

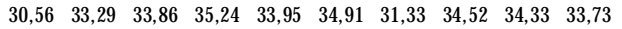
10. Placer $(15,81)(11,66)(12,79)(10,38)(12,16)(11,74)(9,16)(8,65)(8,38)(11,38)$

Fuente: Elaboración propia

Nota: entre paréntesis se anuncian los val ores de desviación típica.

Asimismo, las Tablas 7 y 8 sintetizan los resultados más importantes. La Tabla 7 identifica y recuenta las diferencias estadísticamente significativas en cada variable de perfil, y laTabla 8 muestra el mayor agrado y desagrado de cada uno de los colectivos resultantes según cada variable. Véase que en el caso de las variables de perfil que resultan en más de dos categorías los resultados muestran la categoría donde reside el valor con mayor agrado y mayor desagrado. Así, por ejemplo, se indica para cada valor del cuerpo con diferencias estadísticamente significativas, cuál es la provincia o la modalidad de bachillerato que más tendencia muestra hacia el agrado y cuál hacia el desagrado.

Tabla 7

Valores del cuer po que presentan diferencias estadísticamente significativas según la variable de perfil estudiada.

\begin{tabular}{lccccc}
\hline Variable & Sexo & Modalidad & Naturaleza & Titularidad & Provincia \\
\hline No- Diferencias & $5+(1)$ & 1 & $(2)$ & 1 & $4+(2)$ \\
\hline 1. Biológico & & & & & $(X)$ \\
2. Ecológico & & & & & X \\
3. Instrumental & $\mathrm{X}$ & & & & $\mathrm{X}$ \\
4. Dinámico & $(\mathrm{X})$ & $\mathrm{X}$ & & & $\mathrm{X}$ \\
5. Afectivo-social & $\mathrm{X}$ & & & & $(\mathrm{X})$ \\
6. Ético & $\mathrm{X}$ & & & & \\
7. Estético & $\mathrm{X}$ & & & & $\mathrm{X}$ \\
8. Religioso & $\mathrm{X}$ & & $(\mathrm{X})$ & $\mathrm{X}$ & \\
9. Intelectual & & & $(\mathrm{X})$ & & \\
10. Placer & & & & &
\end{tabular}

10. Placer

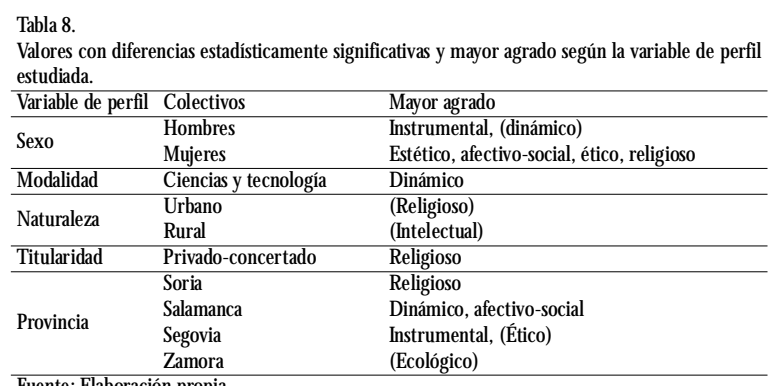

Fuente: Elaboración propia.
Nota: los señalados entre paréntesis refieren diferencias cercanas al valor mínimo para ser consideradas estadísticamente significativas.

Se comprueba cómo la variable sexo registra 5 diferencias estadísticamente significativas y 1 cercana a ser 
considerada como tal, la variable provincia 4 y 2 cerca nas, y la variable naturaleza sólo 2 cercanas. El resto sólo presentan 1 valor con diferencias significativas.

\section{Discusión}

Se han encontrado diferencias significativas en función de las variable sexo y provincia de pertenencia del centro de estudios. Por su parte, el resto de variables tenidas en cuenta, modalidad de bachillerato, naturaleza, y titularidad del centro, sólo han presentado diferencias estadísticamente significativas en alguno de los valores del cuerpo pudiendo llevar a cabo una lectura detallada de cada uno de ellos, pero no pudiendo concluir que dicha variable influye de manera generalizada sobre la formación de los val ores del cuerpo.

El valor religioso es en todo caso un valor controvertido cual quiera que sea la variable de perfil del estudiante tenida en cuenta, sin embargo, no se aborda una interpretación exhaustiva al respecto dado que el objeto de estudio en la presente investigación es el cuerpo y sus valores, y no la tendencia religiosa en cada caso.

De este modo, se puede concluir sólo las variables de perfil sexo y, de manera más dudosa, provincia comportan diferencias significativas en los val ores del cuerpo para la muestra de alumnos estudiada.

\section{Variable sexo del estudiante}

Los valores que presentan diferencias estadísticamente significativas son el valor afectivo-social, estético, ético y religioso, mostrando mayor agra do por parte de las mujeres, así como el valor instrumental con mayor agrado por parte de los hombres. Asimismo, el valor dinámico no llega a presentar diferencias estadísticamente significativas entre hombres y mujeres, pero se sitúa cerca del umbral (0.05).

Finalmente, cinco de los diez valores del cuerpo descritos muestran diferencias estadísticamente significativas entre hombres y mujeres, y uno muy cercano al mínimo requerido.

Los valores estético, afectivo-social y religioso mostrados como más agradables por parte de las mujeres confirman la perspectiva mantenida por la literatura.

En lo que se refiere al valor estético, Ramos, Rivera y Moreno (2010) o Mataix (2012) sostienen que las mujeres se muestran menos satisfechas con su imagen corporal o valor estético. Por su parte, Tubert (1992, 2010) explica que en las mujeres existe una mayor imposición social del valor estético del cuerpo. A este respecto, Guzmán, F. J. B., Fermán, I. A. T., Torres, A. A.
B. \& Díaz, F. J. G. (2005) afirma obtiene que las mujeres muestran mayores puntuaciones en los val ores estéticos.

Y en lo que al valor afectivo-social respecta, se apoyan las conclusiones arrojadas por Álvarez (2002), sobre los valores de la persona, donde las mujeres muestran mayor agrado por losvaloresemoción y sentimiento. Se definen diferencias entre sexos en el valor afectivo, concretamente en dos de los ítems que definen dicha categoría, el valor emoción y el valor sentimiento, siendo las mujeres las que obtienen las mayores puntuaciones. Paralelamente, Naifeh y Smith (1987) y Riso (2003, 2015) argumentan las razones sociales que prohíben la expresión afectivo-social de los hombres.

Así, se comprueba cómo la mujer reporta mayor presión social por la apariencia de su cuerpo, mientras que el hombre se ve reprimido en mayor medida que la mujer a la hora de expresar su emoción y sentimientos.

En cuanto al valor religioso, la literatura sostiene que las mujeres son más religiosas que los hombres (O rozco-Parra, C. L., \& del Carmen Domínguez-Espinosa, A. (2014), Desrosiers, 2011; Miller y Stark, 2002; Walter y Davie, 1998).

Paralelamente, Diaz-Loving, R., Aragón, S. R. , \& Aragón, R. S. (2001) destacan rasgos instrumentales en los hombres y expresivos en mujeres.

\section{Variable modalidad de bachillerato del estu- diante}

En primer lugar, se advierte que la modalidad de artes cuenta con pocos estudiantes en su muestra, 10 cual limita la validez de los resultados arrojados.

En este caso, sólo el valor dinámico presenta diferencias estadísticamente significativas con el mayor agra do por parte de los estudiantes de la modalidad de Ciencias y Tecnología.

Son muchos los estudios que anal izan las relaciones entre nivel de condición física y de práctica físico-deportiva en todos los rangos de edad coincidentes con las diferentes etapas educativas. Centrando la atención sobre la etapa de educación secundaria no obligatoria 0 bachillerato se destacan a autores como Paredes, T. (2016), Narváez, C.J. (2014), Marcelo, A. T. W. (2013) oValdes, P. yYanci, J. (2016). Asimismo,Alonso,T. (2002) compara el nivel de condición física (CF) entre adolescentes de primero de bachillerato y ciclos formativos de grado medio obteniendo mejores resultados de condición física en los alumnos de bachillerato.

Sin embargo, son pocos los estudios que analizan en 
detalle las diferencias y similitudes mantenidas entre los estudiantes de las distintas modalidades de bachillerato. Sebastiá-Amat, S. y otros (2019) concluyen en su estudio que los alumnos de primero de bachillerato de ciencias registran mayores puntuaciones en educación física y los niveles más altos de actividad física. Asumiendo el nivel de práctica física como un indicador observable y cuantificable del valor dinámico del cuerpo, los resultados de dicho estudio son coincidentes con los del presente dado que en ambos casos los alumnos de ciencias parecen mostrar una mayor predisposición al ejercicio físico y su valor dinámico o de movimiento.

\section{Variable naturaleza del centro de estudios}

Se recuerda cómo la variable naturaleza del centro de estudios evocaba su pertenencia a un ámbito rural 0 urbano.

Son los valores del cuerpo religioso e intelectual los más cercanos a al canzar una diferencia estadísticamente significativa, sin embargo, no llega a conseguir la diferencia mínima o representatividad requerida. En el caso del valor religioso es el colectivo de estudiantes pertenecientes a centros de natural eza urbana quienes mayor agrado muestran, mientras que en el valor intelectual se registra mayor agrado en centros rurales.

Así, en términos generales, podría decirse que los valores del cuerpo son muy parecidos en zonas rurales y urbanas, lo que algunos autores podrían explicar por el proceso de globalización y la razonada dificultad de supervivencia de una minoría representada por el contexto social rural frente al influjo de una mayoría urbana que penetra gracias a la televisión como factor más influyente en la Sociedad de la Información y la Comunicación. Según afirma Freire, P. $(1975$, p.50) «las áreas campesinas están siendo al canzadas por lasinfluencias urbanas, a través de la radio, de la comunicación más fácil, por medio de los caminos que disminuyen distancias,》. Finalmente, Bustos (2009) sostiene que se advierte complicado identificar hábitos, actitudes y valores diferenciados entre núcleos rurales y urbanos.

Por su parte, autores como Boix, R. (2003) conceptualizan esta perspectiva como la desrural ización del entorno rural o, como al gunos denominan, zona «suburbana» Gil, M. G. (2011, p. 6) quien toma como referente de medida en esta disonancia el concepto de urbano.

\section{Variable titularidad del centro de estudios}

En primer lugar, recuérdese la connotación asumida para la categoría centros privados-concertados frente a centros públicos. La muestra disponible es escasa para centros privados y también para centros con concierto en bachillerato, por lo cual se decidió aunar ambas categorías bajo una única denominada centros privados-concertados.

En este caso sólo el valor religioso describe diferencias estadísticamente significativas, y lo hace a favor del menor desagrado por parte de los estudiantes de centros privados-concertados que por parte de estudiantes de centrospúblicos. En todo caso, el valor religioso siempre asume connotaciones de desagrado.

Son numerosos los autores que han dedicado sus esfuerzos a relatar, en muchos casos a denunciar, el proceso de secularización de la escuela, situando el punto de inflexión en la segunda república (1931 - 1939). Así, Cárcel, V. (1990) relata la España de la Segunda República y la persecución religiosa, González, B. (2008, 2012), Gómez, F. y González, B. (2015) y Millán, F. (1983) trasladan la repercusión de dicha negación religiosa en la sociedad sobre la práctica pedagógica, es decir, lo que podría llamarse el laicismo escolar a partir de aquel momento.

Como resultado de ello, de dicha revolución laica a nivel social y educativo o de la escuela, podemos comprender la poca consideración del valor del cuerpo religioso mostrada por los estudiantes participantes en el presente estudio, especialmente en los centros públicos.

Este proceso general de secularización de la enseñanza ha sido menos acusado en los centros privadosconcertados que en los centros públicos dada la identidad religiosa por ellos representada. Coincidiendo con esta apreciación, autores como Faubell, V. (2000) y González, T. (2005) relatan las particularidades acaecidas en la educación vinculada a órdenes y congregaciones religiosas y a la escuela pública respectivamente.

\section{Variable provincia del centro de estudios}

Es importante destacar la realidad acontecida en provincias pequeñas como puedan ser Soria, Ávila 0 Zamora. La muestra ha sido seleccionada siguiendo el criterio de proporcionalidad respecto de la población total en todas y cada una de sus categorías, de tal manera que las provincias con escaso número de habitantes han delimitado muestras poco numerosas en el presente estudio empírico. En este sentido, aunque se identifiquen diferencias, éstas deberían ser nuevamente valoradas por su escasa muestra tenida en cuenta.

La variable provincia de pertenencia del centro de estudios comporta diferencias estadísticamente signifi- 
cativas en el valor religioso, dinámico, instrumental y afectivo-social. Asimismo, los valores ético y ecológico anuncian diferencias cercanas a su consideración como estadísticamente significativas. El valor religioso es más agradable para los estudiantes de Soria y menos para los de Valladolid, el valor dinámico es más valorado por alumnos de Salamanca y menos por alumnos de Ávila, el valor instrumental es considerado más agradable por alumnos de Segovia y menos por los de Soria y, finalmente, el valor afectivo-social se registra como más agradable por los estudiantes de Salamanca y menos por los de Soria. Asimismo, los valores del cuerpo ético y ecológico son más agradables para los estudiantes de la provincia de Segovia y menos para los de Zamora.

Asumiendo cada provinciacomo un grupo socio-cultural diferenciado del resto o con varias particularida des comunes a muchos de sus miembros, y al cuerpo como una de las más importantes concepciones socioculturales, los resultados constatan la provincia como un factor importante en la construcción del valor del cuerpo. La provincia es así un factor de influencia con identidad propia a nivel socio-cultural.

Las diferentes provincias que articulan las Comunidad Autónoma de Castilla y León repercuten numerosas diferencias en su contexto. Estas diferencias son ampliamente detalladas por estudios a nivel político, económico, médico, de recursos, etc. Estas diferencias de contexto entre provincias, forman parte de la ecuación que define la identidad social de dicho territorio. Siguiendo a Giménez, G. (2010) en consonancia con Izzo (1985), las identidades se inscriben y apoyan en un contexto de representaciones sociales compartidas, es decir, tradiciones culturales, expectativas recíprocas, saberes compartidos y esquemas comunes. Giménez, $\mathrm{G}$. explica ampliamente las relaciones en el binomio identidad-cultura como pareja conceptual indisociable.

Por su parte, Inglehart (1991) sostiene que son las condiciones sociales en las que los jóvenes se crían y educan las que configuran prioritariamente su sistema de valores. Los valores son inherentes a cual quier realidad y la primera de ellas, no es sino el cuerpo. Esta primera dimensión de la persona que le da acceso al mundo es quizás la esencia más tatuada por la sociedad, la que en mayor medida recoge los estereotipos e idiosincrasia de la cultura que representa. Barbero (2007, p. 26) afirma que «el capital corporal forma parte del amplio y diverso conjunto de capitales culturales». A este respecto, Baudrillard (2009) afirmaqueel lugar que ocupa el cuerpo es un hecho de cultura, ya que en cualquier cultura supone el modo de organización de la relación con las cosas y el modo de organización de las relaciones socia les. Por su parte, Rodolfo Rozengardt (2013) advierte que cualquier reflexión del cuerpo debe contemplar y, al mismo tiempo aunar, sus dimensiones y consideraciones de orden histórico, político, epistemológico y pedagógico. A las cuales debemos añadir las biológicas y psicológicas. La afirmación de Rozengardt (2013) abre todo un discurso sobre el binomio cuerpo-cultura seña lando que el cuerpo humano sin la cultura es una abstracción biologista o psicologista, pero cultura sin cuerpo es directamente una afirmación imposible.

Tras el estudio detallado de la influencia de las variables de perfil de los estudiantes en los valores del cuerpo de la sociedad, se sugiere una gran importancia de determinadas características del individuo en la determinación de sus valores. De este modo, se advierte que a la hora de intervenir en educación es imprescindible no sólo describir generalidades de los estudiantes, sino también sus particularidades asociadas a sus variables de perfil para poder adaptar en mayor medida la educación a sus características particulares.

\section{Conclusiones}

Los resultados demuestran que la variable sexo es indiscutiblemente un factor de influencia en la construcción de valores en la medida que presenta diferencias estadísticamente significativas en 5 de los 10 valores del cuerpo y 1 cercanas a serlo. Por su parte, la variable provincia de pertenencia del centro de estudios también presenta 4 valores con diferencias estadísticamente significativas y 2 cercanas a ello, por lo que puede ser considerado como un segundo factor de influencia en la formación de valores. Sin embargo, el resto de variables de perfil consideradas, modalidad de bachillerato cursada, naturaleza y titularidad del centro de estudios no comportan diferencias 0 estadísticamente significativas en la mayoría de valores del cuerpo, por lo que se puede concluir que no se presentan en este caso, como un determinante realmente influyente en la formación de los valores del cuerpo de los estudiantes de bachillerato. Si bien, estas variables son para algunos de los valores del cuerpo en concreto realmente poderosas.

Emerge, así, el reto educativo de reformular y rehabilitar el cuerpo cada día de su existencia. El docente, como principal responsable en esta labor de guiar al alumno hacia su propio yo, puede partir de la lectura de la realidad que ofrece el presente artículo, haciendo a sus discentes recapacitar sobre lo que podría ser fruto 
de la tendencia social, de la naturaleza, o de su propia construcción personal.

En este sentido apuntamos la necesidad de una política curricular, especialmente activa en la disciplina de educación física, vigilante sobre la imagen del cuerpo no alienante (Bernard, 2006:141), garantizando el derecho a ser diferentes e incluso marginales (Denis 1980:180). Una reconsideración de la metodología empleada en educación física más proclive a una participación activa en el proceso de enseñanza-aprendizaje, con mayor protagonismo del alumno, pleno partícipe en el proceso finalmente educativo. Asimismo, una metodología menos individualista que enriquezca las relaciones sociales y cree valor del encuentro corporal. Se torna así especialmente útil el aprendizaje coopera tivo puesto que el valor es inherente a cualquier experiencia y el contacto humano emergente de una rela ción social es una experiencia también corporal. Recordando que, como abría la introducción, el cuerpo especialmente en la adolescencia, es más un producto social quepsico-fisiológico.

\section{Referencias}

Álvarez Rodríguez, J. (2002). Análisis descriptivo de los valores sentimiento y emoción en la formación de profesores de la Universidad de Granada. Profe sorado: Revista de curriculum y formación del profesorado, 6(1).

Álvarez Rodríguez, J., Rodríguez Sabiote, C., y Lorenzo Q uiles, O. (2007). Investigación sobre los valores de los jóvenes universitarios españoles. Un estudio comparado. Revista Internacional de Ciencias Sociales y H umanidades, SO CIOTAM , XVII (1), 25-46.

Barbero, J. I. (2007). Capital(es) corporal(es) que configuran las corrientes y/ o contenidos de la educación física escolar. Ágora para la educación física y el deporte, (4), 21-38.

Baudrillard, J. (2009). La sociedad de consumo: sus mitos, sus estructuras. Madrid: Siglo XXI.

Boix Tomàs, R., Roser (1998). «La educación primaria en el medio rural español». Revista Aula de Innovación Educativa, 77, 43-46.

Bustos Jiménez, A. (2009). «Valoraciones del profesorado de escuela rural sobre el entorno presente». Revista Iberoamericana de Educación, 48/ 6 .

Cámara Estrella, A. M. (2010). Val ores en futuros profesores de Secundaria. Revista electrónica interuniversitaria deformación del profesorado, 13(3), 113121.
Cárcel O rtí, V. (1990). La persecución religiosa en España durante la Segunda República (1931-1939), Madrid: Ediciones Rialp, S.A,

Casares García, P. M. (1995). Test de valores: un instrumento para la evaluación. Revista española de pedagogía, 53(202), 513-537.

Casares García, P. M . , y ColladosZorraquino, J. (1998). Evaluación de valores del cuerpo educando. Revista de ciencias de la educación: Organo del Instituto Calasanz de Ciencias de la Educación, (174), 237-258.

Desrosiers, A. (2011). Relational spirituality in adolescents: Exploring associations with demographics, parenting style, religiosity, and psychopathology (Tesis doctoral).

Diaz-Loving, R., Aragón, S. R., \& Aragón, R. S. (2001). Rasgos instrumentales (masculinos) y expresivos (femeninos). Revista latinoamericana de Psicología, 33(2), I3|-I39.

Faubell Zapata, V. (2000). Educación y órdenes y congregaciones religiosas en la España del siglo XX, Revista de Educación, no extr., 137-200.

Fize M. ¿Adolescencia en crisis? Por el derecho al reconocimiento social (2004). Madrid: Siglo XXI Editores S.A.

Freire, P (1975). ¿Extensión o comunicación? La concientización en el medio rural. Argentina, Buenos Aires: Siglo XXI.

Gil, M. G. (2011). La escuela de contexto rural: ¿de la diferencia a la desigualdad? Revista Iberoamericana de Educación, (55/ 5)

Giménez, G. (2010). Cultura, Identidad y procesos de individualización. Conceptos y fenómenos fundamentales de nuestro tiempo. U niversidad N acional Autónoma de México.

Gómez, F. P. \& \& González, B. H. (2015). Escuelay renovación pedagógica en España: una mirada histórica. Escenarios, 1(17), 85-100.

González, B. H. (2012). La práctica educativa laica en la escuela pública española (1931-1939) (Doctoral dissertation, Universidad de Alcalá).

Gonzál ez, B. H. (2008). Laicismo escolar en la Segunda República española. I Encuentro de Jóvenes Investigadores en Historia Contemporánea de la Asociación de H istoria Contemporánea: Zaragoza, 26, 27 y 28 de septiembrede 2007 (p. 65). Prensas U niversitarias de Zaragoza.

González Vila, T. (2005). Laicidad, libertad religiosa y escuela pública. Religión y escuela: la revista del profesorado de religión, 189, 20.

Guzmán, F. J. B., Fermán, I. A. T., Torres, A. A. B., \& Díaz, F. J. G. (2005). Un estudio comparativo sobre 
valores éticos en estudiantes universitarios. Enseñanza e Investigación en psicología, 10(2), 397-415.

Millán, F. (1983). La Revolución Laica de la Institución Libre de Enseñanza a la Escuela de la República. Valencia: Fernando Torres-Editor.

Miller, A. y Stark, R. (2002). Gender and religiousness: Can socialization explanations be saved? American Journal of Sociology, 107(6), 1399-1423.

García-Caballero C. (2000). Tratado de Pediatría Social. Madrid: Ediciones Díaz de Santos S.L.

Gervilla Castillo, E. (2000). Un modelo axiológico de educación integral. Revista española de pedagogía, 3957.

Gervilla Castillo, E. (2000). Valores del cuerpo educando: antropol ogía del cuerpo y educación. Barcelona: Herder.

Inglehart, R. (1991). El cambio cultural en las sociedades industriales avanzadas. Madrid: Centro de investiga ciones sociológicas.

Izzo, A. (1983). II concetto di mondo vitale. AA. VV.»Complessità sociale ed identità», Franco Angeli, Milano.

Marcelo, A. F. W. (2013). Análisis de la condición física de

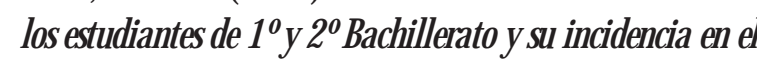
rendimiento deportivo del colegio universitario en el año 2012/ 13 (Tesis Doctoral, U niversidad Técnica del Norte).

Mataix, J. (2012). Culto al cuerpo: ¿cuál es el precio de la belleza? Actas D ermosifiliográficas, 103(8), 655-60.

Naifeh, S., y Smith, G. W. (1987). ¿Por qué los hombres ocultan sus sentimientos? Javier Vergara.

Narváez Anrango, C. J. (2014). Estudio de la condición física y su incidencia en el entrenamiento de las carreras de medio fondo de los estudiantes del segundo año de bachillerato del colegio nacional San Pablo de la parroquia de San Pablo del Lago en el año lectivo 2012-2013 (Bachelor's thesis).

Paredes Gómez, R. A. (2016). Estudio de la condición física de los estudiantes del 3 nivel de bachillerato de la unidad educativa Jacinto Collahuazo dela ciudad de Otavalo en el periodo 2015-2016 (Bachelor's thesis).

Q uijano Magaña, D., Lorenzo Q uiles, 0., y Alegre Benítez, C. (2016). Elección y preferencia de valores en estudiantes universitarios mexicanos. Profesorado, Revista de Currículum y Formación del Profesorado, 20(2), 18-38.

Q uijano Magaña, D., y Lorenzo Q uiles, O. (dir. tes. (2015, septiembre 4). Percepciones sobre valores en estudiantes universitarios del estado deYucatán.

O rozco-Parra, C. L., \& del Carmen Domínguez-Espinosa, A. (2014). Diseño y validación de la Escala de Actitud Religiosa. Revista de Psicología, 23(1), 3-11

Riso, W. (2003). Intimidades mascul inas: sobre el mito de la fortaleza masculina y la supuesta incapacidad de los hombres para amar. Barcelona: Editorial Norma.

Riso, W. (2015). Lo que toda mujer debe saber acerca de los hombres: la afectividad masculina. México: Editorial 0 ceano.

Rozengardt, R. (2013). La Educación Físicay el cuerpo en la escuela. En X Congreso Argentino yV Latinoamericano de Educación Física y Ciencias.

Sebastiá-Amat, S., GarcíaJaén, M., Ferriz Valero, A., \& Jimenez-O Imedo, J. M. (2019). Relación entrela actividad física, la educación física y el rendimiento académico en el alumnado de bachillerato. Revista Iberoamericana de Psicología del ejercicio y el Deporte.Vol. $14, n-2$.

Tubert, S. (1992). Desórdenes del cuerpo. Revista de Occidente, (134), 137-153.

Tubert, S. (2010). Los ideales culturales de la feminidad y sus efectos sobre el cuerpo de las mujeres. Q uaderns de Psicología, 12 (2), 161-174.

Valdes, P., \& Yanci, J. (2016). Análisis de la condición física, tipo de actividad física realizada y rendimiento académico en estudiantes de educación secundaria. Retos. Nuevas tendencias en educación física, deporte y recreación, (30), 64-69.

Walter, T. y Davie, G. (1998). The religiosity of women in the modernW est. The British Journal of Sociology, 49(4), 640-660.
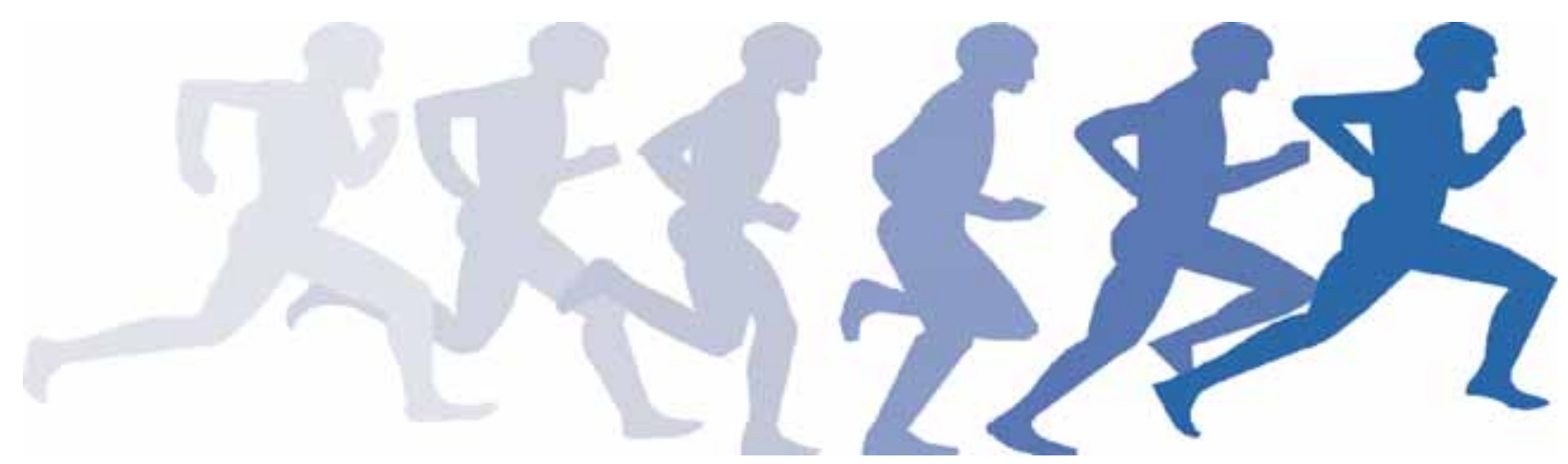